\title{
Hat der Gehalt des Weizenmehles an wasserlöslichem Stickstoff einen Einfluß auf seinen Backwert?
}

\author{
Von \\ W. Bremer. \\ Matteilung aus dem Laboratorium der Hofmühle Dresden-Plauen. \\ (Inhaber: Th. und E. Bienert.)
}

Die Versuche, den Backwert des Weizenmehles aus seinem Klebergehalte abzuleiten, sind nicht immer von dem gewünschten Erfolge begleitet gewesen, doch kann nach den neueren Untersuchungen, namentlich von $\mathrm{Kosutány}{ }^{1}$ ) und $W$. Schneidewind ${ }^{2}$, nicht mehr daran gezweifelt werden, daß der Kleber des Weizenmehles auf die Gebäckausbeute wie auf die Güte des Gebäckes von entscheidendem Einfluß ist, doch gehen über das Wie die Ansichten weit auseinander. Wer sich selbst längere Zeit und eingehend mit dem Klebergehalte des Weizenmehles im Vergleich zu seinem Backwert beschäftigt hat, begreift nicht, wie der Zusammenhang zwischen Backwert und Klebergehalt des Weizenmehles je in Abrede gestellt werden konnte. Immerhin aber mußte man darauf Bedacht nehmen, noch weitere Kennzeichen zu ermitteln, um ein einigermaßen sicheres Verfahren zur Bestimmung des Backwertes der Mehle begründen zu können und so von dem als unbequem empfundenen Backversuche loszukommen. Außer dem Kleber und den wasserunlöslichen Eiweißstoffen könnte für die Beurteilung des Weizenmehles die Stärke in Betracht kommen. Versuche, welche darauf abzielen, die verschieden backfäbigen Mehle auf Grund der Eigenschaften ibrer Stärke unterscheiden zu wollen, können aber von vornherein nicht als sehr aussichtsvoll bezeichnet werden, denn es ist eine bekannte Tatsache, daß die Weizenstärke von der des Roggens nur schwierig zu unterscheiden ist. Das sicherste Kennzeichen bietet noch die Verkleisterungstemperatur ${ }^{3}$ ). Während Roggenstärke in Wasser von $62,5^{\circ}$ schon fast völlig verquillt, behalten die Stärkekörner des Weizens beim Erhitzen bis zu dieser. Temperatur ihre Struktur noch deutlich erkennbar bei, Nun ist es aber nicht weniger bekannt, daß aus Roggenmehl kein dem Weizengebäck auch nur ähnliches Brot bereitet werden kann. Die Gebäcke aus Weizenmehl sind von denen aus Roggenmehl grundverschieden. Bereitet nun schon die Unterscheidung der Stärke zweier so verschiedener Mehlarten nicht unbedentende Schwierigkeiten, so dürfte es völlig unmöglich sein, die einzelnen Spielarten der betreffenden Getreide mit Hilfe ihrer Stärkekörner zu unterscheiden. Vom Fettgehalt des Mehles ist ebenfalls kein entscheidender Einfluß auf das Gelingen des Gebäckes zu erwarten, da die Hefen und 329.$)$

1) Kosutány, Über Weizen und Weizenmehl. (Journ. f. Landwirtsch. 1905, 51, 139

2) W. Schneidewind: Arbeiten aus der Landwirtschaftskammer der Prov. Sachsen Heft II and Landw. Jahrbücher 1904, 38, 269.

3) J. König, Untersuchung landwirtschaftlich und gewerblich wichtiger Stoffe. Berlin 1906, S. 293. 
das Fett zunächst gar nicht angreifen, sondern sich lediglich die Kohlenhydrate zu nutze machen. Außerdem enthält aber nur der Keim des Weizens beachtenswerte Mengen Fett, und da nach dem heutigen Mahlverfahren vom Keim nur geringe Spuren mit in das Mehl gelangen, so ist im fertigen Verkaufsmehl nicht mehr als $1-2 \%$ Fett anzutreffen.

Der Einfluß, den die wasserlöslichen Stickstoff-Substanzen, Nichtkleberstoffe, auf die Gebäckausbeute und die Güte des Gebäcks ausüben, ist meines Wissens noch nicht näher untersucht worten, weshalb ich es für angezeigt hielt, hierüber einiges Zahlenmaterial zu sammeln. Die Menge der wasserlöslichen StickstoffSubstanz ist zwar keine größere als die des Fettes, aber die Hefe braucht, um einen gut gelockerten Teig zu hinterlassen, zweifellos lösliche Stickstoffverbindungen, da ohne solche eine üppige Sprossung der Hefe ausgeschlossen ist. Nach meinen Beobachtungen erleidet nun der Kleber durch die Hefengärung so gut wie keine Veränderung. Auf die äußere Beschaffenheit des Klebers ist es völlig ohne Einfluß, ob er frischem, nur mit Wasser angerührtem Teige entstammt, oder ob er aus fertigem, ofengarem Teige gewonnen ist, der unter entsprechendem Kochsalzzusatze bereitet warde und von Hefe durchgoren ist. Um so mehr erschien es mir daher wünschenswert, zu erfahren, ob die wasserlöslichen Stickstoffverbindungen einen Einfluß auf das Gelingen des Gebäcks ausüben oder nicht.

Die Untersuchungen wurden nur mit ganzen Mehlen vorgenommen. Sämtliche Mehle sind aus Weizen 1906-er Ernte gewonnen und von den nachfolgenden Herkünften :

1. Deutsche Weizen. a) Sächsische. b) Preußische.

2. Donau-Weizen (Balkanstaaten).

3. Russische Weizen. a) Podolisch-Wolhynisch-Polnische Weizen. b) Südrussische Weizen.

4. Nordamerikanische Weizen.

5. Südamerikanische Weizen.

Über die Versuchsanordnung ist folgendes mitzuteilen: $20 \mathrm{~g}$ Mehl wurden mit $12 \mathrm{ccm}$ Brunnenwasser eingeteigt. Der Teig blieb in einem Porzellansckälchen, mit einer Glastafel bedeckt, bei Zimmertemperatur 1/2 Stunde sich selbst überlassen. Alsdann wurde der Teig mit Brunnenwasser von etwa $17^{\circ} \mathrm{C}$ ausgewaschen, wobei mit Hilfe eines Trichters mit großer Offnung alles ablaufende Wasser in einem Kolben aufgefangen wurde. Bei einiger Vorsicht gelingt es leicht, das Wasser verlustlos aufzufangen. Die Flüssigkeit wurde alsdann zu $500 \mathrm{ccm}$ aufgefüllt und nach dem Umschütteln und Absitzenlassen der Stärke wurden von der darüber stehenden, fast klaren Lösung 100 bezw. $50 \mathrm{ccm}$ entsprechend 4 bezw. $2 \mathrm{~g}$ Mehl abpipettiert und nach Zusatz einiger Tropfen Schwefelsäure über freier Flamme bis auf $15-20 \mathrm{~cm}$ eingeengt. Die auf diese Weise erhaltene Flüssigkeit wurde nach Überspülung in einen $\mathrm{Kjeldahl-Kolben} \mathrm{in} \mathrm{der}$ bekannten Weise mit Schwefelsäure und Quecksilber verbrannt. Eine Korrektur für das Volumen der Stärke im $500 \mathrm{cem}-$ Meßkolben fand nicht statt; doch dürfte der hierdurch bewirkte Fehler verschwindend klein sein, sodaß er außer Betracht bleiben kann.

Da nach Kosutány's Ansicht die Beschaffenheit des Teiges und Klebers auf das Gelingen des Gebäcks nicht ohne Einfluß ist, so habe ich auch die leicht wahrnehmbaren physikalischen Eigenschaften des Klebers und Teiges aufgezeichnet. Zu 
einer Messung der Festigkeit des Teiges mit dem Reitö'schen Festigkeitsprüfer bin ich noch nicht gekommen, jedoch hoffe ich, in einer späteren Arbeit hierüber berichten zu können. Nach meinen bis jetzt an reichlich 300 Proben verschiedenster Herkunft gemachten Beobachtungen kann man die mit $60 \%$ Wasserzusatz hergestellten Teige hinsichtlich ihrer Festigkeit einteilen in: 1. sehr weiche, fast zerfliebliche, 2. weiche, 3. mittelfeste und 4. feste Teige.

Die sehr weichen und festen Teige besitzen kaurn eine Dehnbarkeit; die weichen und mittelfesten Teige dagegen sind leidlich dehnbar. Während die festen Teige sich so trocken anfühlen, daß sie gar nicht an der Hand Kleben bleiben, hat man bei den stark klebenden mittelfesten und weichen Teigen oft Mühe, dieselben verlustlos von der Hand wieder abzubringen.

War das ablaufende Waschwasser ganz klar, und zeigten sich keine Spuren von Stärke melr, so wurde die Auswaschung als beendet angesehen und der resultierende Kleber auf seine physikalischen Eigenschaften geprüft.

Bei der Beschaffenheit des Klebers ist mir aufgefallen, daß einzelne Weizen, z. B. der Kansas-Weizen, meist einen Kleber zeigen, dem unmittelbar nach dem Auswaschen jede Dehnbarkeit abgeht. Da sich solcher Kleber sehr kurz, ähnlich wie Quark, anfühlt, so wählte ich für diese Art von Kleber die Bezeichnung ,brüchig und kurz". Ich glaube, daß der Kleber mit diesen Eigenschaften dem Fleurent'schen trockenen und spröden Kleber entspricht. Fraglich erscheint mir dagegen, ob Kosután y's "kurzer" Kleber mit demjenigen identisch ist, welchen ich brüchig und kurz nannte, da letzterer nach meinen Beobachtungen eine sehr gute Backfähigkeit des Mehles bedingt, während Kosutány entgegengesetzter Meinung ist. Ich will noch bemerken, daß brüchiger und kurzer Kleber im Boland'schen Aleurometer meist einen geringen Trieb zeigt, sodaß der Metallstempel wenig über $100 \mathrm{~mm}$ aus der Kapsel hervorgetrieben wird.

Dem brüchigen und kurzen Kleber steht eine andere grundverschiedene Art gegenüber, bei welcher ich unmittelbar nach beendetem Auswaschen das Gefühl hatte, als suche er sein Volumen zu vergrößern. Die Oberfläche dieses Klebers ist von Anfang an glatt, was beim brüchigen und kurzen Kleber erst nach einiger Zeit eintritt.

Kleber, wie der zuletzt beschriebene, zeigt im Aleurometer bei $200^{\circ} \mathrm{C}$ einen weit energischeren Trieb, sodal dieser oft mit einem $150 \mathrm{~mm}$ langen Stempel nicht gemessen werden kann, und besitzt eine gute Dehnbarkeit. Die Bezeichnung „plastisch und voll" erschien mir für solchen Kleber am geeignetsten. Zwisehen dem erst beschriebenen Kleber und dem mit „plastisch und voll“ bezeichneten liegen noch Übergangsformen, welche mehr oder weniger nach der einen oder der anderen Art hinneigen.

Nach diesen erläuternden Vorbemerkungen seien die Beobachtungen an den untersuchten 24 Proben mitgeteilt, jedoch will ich noch bemerken, daß zur Bestimmung des Triebes im Aleurometer stets der aus $25 \mathrm{~g}$ Mehl gewonnene Kleber verwendet wurde. Die Wägung des Klebers erfolgte im feuchten Zustande, nachdem derselbe unmittelbar nach dem Auswaschen einheitlich aber nur oberflächlich mit der Hand abgetrocknet worden war. Um auch das Gewicht der Brötchen mit ihrer Größe vergleichen zu können, wurden alle Brötchen bei gleicher Teigeinlage hergestellt und ihre Größen auf je $100 \mathrm{~g}$ der fertigen Gebäcke bezogen. 
I. Deutsche Weizen.

a) Säehsische Weizen.

\begin{tabular}{|c|c|c|c|c|c|c|c|c|c|}
\hline \multirow{2}{*}{ No. } & \multirow{2}{*}{$\begin{array}{c}\text { Art } \\
\text { des Weizens }\end{array}$} & \multirow{2}{*}{$\begin{array}{c}\text { Hekto- } \\
\text { liter- } \\
\text { Gewicht } \\
\mathrm{kg}\end{array}$} & \multirow{2}{*}{$\begin{array}{c}\text { Kleber- } \\
\text { menge } \\
0_{i 0}\end{array}$} & \multicolumn{2}{|c|}{ Beschaffenheit des } & \multicolumn{2}{|c|}{$\begin{array}{c}\text { Triebkraft } \\
\text { des Klebers }\end{array}$} & \multirow{2}{*}{\begin{tabular}{|c|} 
Wasser \\
10sliche \\
Stiek- \\
stoff- \\
Sub- \\
stanz \\
$\%$ \\
$\%$
\end{tabular}} & \multirow{2}{*}{$\begin{array}{l}\text { Volu- } \\
\text { men } \\
\text { ron } \\
100 \mathrm{~g} \\
\text { grobäck } \\
\text { cem }\end{array}$} \\
\hline & & & & Teiges & Ilebers & $\begin{array}{c}\text { Menge } \\
\mathrm{g}\end{array}$ & höhe & & \\
\hline 1 & Landweizen & 77,5 & 35,2 & Wenig klebend & $\begin{array}{c}\text { Anfangs sehr weich, } \\
\text { später plastiseh } \\
\text { und voll }\end{array}$ & 8,8 & 92 & 1,14 & 309,8 \\
\hline 2 & Sommerweizen & 78,0 & 45,6 & weich und klebend & plastisch und voll & 11,4 & 150 & 1,05 & 422,2 \\
\hline
\end{tabular}

b) Preufische Weizen.

\begin{tabular}{|c|c|c|c|c|c|c|c|c|c|}
\hline 3 & $\begin{array}{c}\text { Kolben- } \\
\text { Sommerwoizen }\end{array}$ & 78,5 & 49,6 & $\begin{array}{l}\text { senx weich bnd } \\
\text { qfemlith klebend }\end{array}$ & briehig nnd karz & 12,4 & 150 & 1,54 & 418,4 \\
\hline 4 & Desgl. & 78,5 & 42,0 & $\begin{array}{l}\text { etwas klebend, } \\
\text { mittelfest; betm } \\
\text { Auswaschen hart }\end{array}$ & $\begin{array}{l}\text { gentr brüehig } \\
\text { und kurz }\end{array}$ & 10,5 & 110 & 1,64 & 428,2 \\
\hline 5 & $\begin{array}{c}\text { Noë- } \\
\text { Sommerweizen }\end{array}$ & 79,0 & 42,8 & $\begin{array}{l}\text { klebend, } \\
\text { mittelfest }\end{array}$ & $\begin{array}{c}\text { plastisuh, leidlich } \\
\text { dehnbar; beim Aus- } \\
\text { waschen etwas } \\
\text { weich }\end{array}$ & 10,7 & 150 & 1,17 & 325,6 \\
\hline 6 & Weißs-Weiren & - & 28,4 & $\begin{array}{l}\text { weieh und klebend; } \\
\text { beim Auswaschen } \\
\text { fast zerfliebend }\end{array}$ & $\begin{array}{l}\text { nicht dehnbar, } \\
\text { etwas weich }\end{array}$ & 7,1 & 75 & 0,90 & 382,4 \\
\hline 7 & $\begin{array}{c}\text { Sehlesischer } \\
\text { Sommerweizen }\end{array}$ & 80,0 & $44: 4$ & $\begin{array}{l}\text { klebend, } \\
\text { mittelfest }\end{array}$ & $\begin{array}{l}\text { wenig dehnbar, } \\
\text { etwas weich }\end{array}$ & 11,1 & 145 & 1,05 & 397,3 \\
\hline
\end{tabular}

\section{Donau-Weizen.}

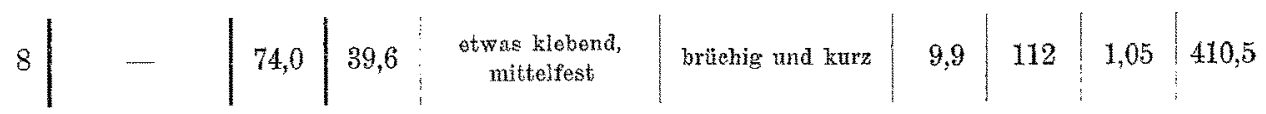

\section{Rassische Weizen.}

a) Podolisch-Wolhynisch-Polnische Weizen.

\begin{tabular}{|c|c|c|c|c|c|c|c|c|c|}
\hline 9 & $\begin{array}{l}\text { Ohne nühers } \\
\text { Bezeichnung }\end{array}$ & 76,5 & 44,0 & $\begin{array}{l}\text { wenig Hlebend, } \\
\text { mittelifest; beim } \\
\text { Auswaschen etwras } \\
\text { zerfliebend }\end{array}$ & brüchig und kurz & 11,0 & 150 & 1,46 & 408,7 \\
\hline 10 & Desgl. & 74,5 & 47,2 & $\begin{array}{l}\text { ziemlieh weich } \\
\text { und klebend }\end{array}$ & $\begin{array}{l}\text { plastisch und voll, } \\
\text { aber nieht dehnbar }\end{array}$ & 11,8 & $\begin{array}{l}\text { Weit } \\
\text { uiber } \\
150\end{array}$ & 0,93 & 465,1 \\
\hline 11 & Desgl. & 75,5 & 40,8 & $\begin{array}{l}\text { sehr" weich } \\
\text { und klebend }\end{array}$ & $\begin{array}{l}\text { nicht dehnbar, } \\
\text { aber weich }\end{array}$ & 10,2 & 135 & 1,00 & 508,5 \\
\hline 12 & $\begin{array}{l}\text { Polniseher } \\
\text { Buntweizen }\end{array}$ & 75,0 & 40,4 & $\begin{array}{l}\text { weich und ziemlich } \\
\text { klebend }\end{array}$ & $\begin{array}{l}\text { plastiseh, } \\
\text { leidlieh dehnbar }\end{array}$ & 10,1 & 185 & 1,08 & 416,9 \\
\hline
\end{tabular}

\section{b) Südrussische Weizen.}

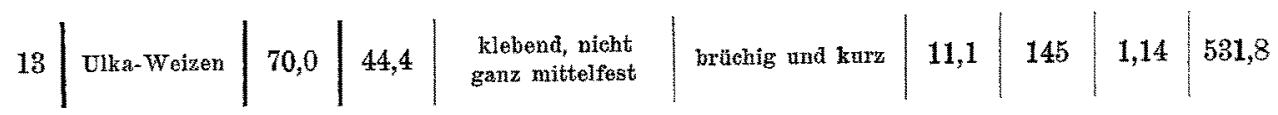




\begin{tabular}{|c|c|c|c|c|c|c|c|c|c|}
\hline \multirow[b]{2}{*}{ No. } & \multirow{2}{*}{$\begin{array}{c}\text { Art } \\
\text { des Weizens }\end{array}$} & \multirow{2}{*}{$\begin{array}{c}\text { Hekto- } \\
\text { liter- } \\
\text { Gewicht } \\
\mathrm{kg}\end{array}$} & \multirow{2}{*}{$\begin{array}{c}\text { Kleber- } \\
\text { menge }\end{array}$} & \multicolumn{2}{|c|}{ Beschaffenheit des } & \multicolumn{2}{|c|}{$\begin{array}{c}\text { Triebkraft } \\
\text { des Klebers }\end{array}$} & \multirow{2}{*}{$\begin{array}{l}\text { Wasser- } \\
\text { lösliche } \\
\text { Stiek- } \\
\text { stoff- } \\
\text { Sub- } \\
\text { stanz } \\
\theta_{0}\end{array}$} & \multirow{2}{*}{$\begin{array}{c}\text { Volu- } \\
\text { men } \\
\text { von } \\
100 \mathrm{~g} \\
\text { Gebäek } \\
\mathrm{g}\end{array}$} \\
\hline & & & & Teiges & Klebers & $\begin{array}{c}\text { angew. } \\
\text { Menge } \\
\mathrm{g}\end{array}$ & $\begin{array}{l}\text { Trieb- } \\
\text { höhe } \\
\text { nmm }\end{array}$ & & \\
\hline 14 & Desgl. & 71,5 & 50,4 & $\begin{array}{l}\text { tiemlich weich } \\
\text { und klebend }\end{array}$ & $\begin{array}{l}\text { brüchig nud lurz, } \\
\text { dabei fest }\end{array}$ & 12,6 & $\begin{array}{l}\text { weit } \\
\text { über } \\
150\end{array}$ & 1,23 & 518,5 \\
\hline 15 & Azima-Weizen & 75,5 & 52,8 & $\begin{array}{l}\text { Kklebend, } \\
\text { mittelfest }\end{array}$ & $\begin{array}{l}\text { wenig dehnbar, } \\
\text { etwas schleimig } \\
\text { und woieh }\end{array}$ & 13,2 & 140 & 1,40 & 599,1 \\
\hline 16 & Desgl. & 74,5 & 49,6 & $\begin{array}{l}\text { klebend, nicht } \\
\text { ganz mittelfest }\end{array}$ & $\begin{array}{l}\text { plastiseh und voll, } \\
\text { leidlieh dehnbar }\end{array}$ & 12,4 & 150 & 1,19 & 498,4 \\
\hline
\end{tabular}

IV. Noxdamerikanische Weizen.

\begin{tabular}{|c|c|c|c|c|c|c|c|c|c|}
\hline 17 & Duluth I & 77,0 & 36,8 & $\begin{array}{l}\text { trocken, } \\
\text { nicht klebend, } \\
\text { ziemlich fest }\end{array}$ & brüchig und kurz & 9,2 & 85 & 1,27 & 421,2 \\
\hline 18 & Hardwinter I & 78,5 & 29,2 & $\begin{array}{l}\text { wenig klebend, } \\
\text { fest }\end{array}$ & desgl. & 7,3 & 95 & 1,10 & 353,7 \\
\hline 19 & Desgl. & 82,0 & 29,2 & $\begin{array}{l}\text { nicht klebend, } \\
\text { trouken und fest }\end{array}$ & $\begin{array}{c}\text { zuerst sehr brüehig } \\
\text { und kkurz, dann sehr } \\
\text { klebend }\end{array}$ & 7,3 & 55 & 1,24 & $\left\{\begin{array}{l}363,0 \\
382,0\end{array}\right.$ \\
\hline 20 & Desgi. & 80,0 & 31,2 & $\begin{array}{c}\text { mittelfest, } \\
\text { nicht klebend }\end{array}$ & brütehig und kurz & 7,8 & 60 & 0,83 & 370,5 \\
\hline 21 & Kansas & 79,0 & 31,2 & $\begin{array}{c}\text { mittelfest, } \\
\text { wenig klebend }\end{array}$ & desgl. & 7,8 & 95 & 1,19 & 412,1 \\
\hline 22 & Hardwinter I & 77,5 & 33,2 & $\begin{array}{c}\text { mittelieat; sehr } \\
\text { wenig tlebend }\end{array}$ & desgl. & 8,3 & 84 & 0,92 & 444,2 \\
\hline
\end{tabular}

V. Südamerikanische Weizen.

\begin{tabular}{c|c|c|c|c|c|c|c|c|c}
23 & $\begin{array}{c}\text { La plata- } \\
\text { Weizen }\end{array}$ & 77,5 & 34,4 & $\begin{array}{c}\text { nicht sehr fest, } \\
\text { klebend und } \\
\text { dehnbar }\end{array}$ & $\begin{array}{c}\text { anfangs weich, } \\
\text { dann brijehig und } \\
\text { kurz }\end{array}$ & 8,6 & 82 & 1,22 & 305,5 \\
\hline 24 & $\begin{array}{c}\text { Rosario } \\
\text { Savta Fó }\end{array}$ & 76,5 & 41,2 & $\begin{array}{c}\text { ziemlich weieh } \\
\text { und klebend }\end{array}$ & $\begin{array}{c}\text { sehr brtichig } \\
\text { und kurz }\end{array}$ & $\mathbf{1 0 , 3}$ & 112 & 1,23 & $\mathbf{4 6 0 , 8}$
\end{tabular}

Nach den vorstehend mitgeteilten Zahlen muß es mindestens zweifelhaft erscheinen, ob der wasserlösliche Stickstoff einen Einfluß auf die Backfähigkeit des Mehles ausübt. Die höchste Gebäckausbeute betrug $599,1 \mathrm{ccm}$ für $100 \mathrm{~g}$ Gebäck. Das zugehörige Mehl aus südrussischem Azima-Weizen enthielt 1,40 ${ }_{10}^{\prime}$ Stickstoff-Substanz in wasserlöslicher Form. Das geringste Gebäck-Volumen von $305^{1 / 2} \mathrm{ccm}$ für $100 \mathrm{~g}$ wurde bei einem Mehle aus La Plata-Weizen erzielt, welches 1,22\% wasserlösliche Stickstoff-Substanz enthielt. Der Unterschied von $0,18 \%$ im Gehalt an wasserlöslicher Stickstoff-Substanz ist zu unbedeutend, um einen solchen auffallenden Unterschied in der Gebäckausbeute bedingen zu können. Vielleicht spielt aber in dieser Hinsicht nicht die Gesamtmenge der löslichen Stickstoff-Substanzen eine Rolle, sondern ist die Backfähigkeit von bestimmten wasserlöslichen Stickstoff-Verbindungen oder sonstigen wasserlöslichen Körpern abhängig. 
Insofern erscheint mir das Dunkel, welches über der Backfähigkeit des Weizenmehles lagerte, gelichtet zu sein, als der Kleber für die Bewertung des Mehles in erster Linie in Betracht zu ziehen ist. Unter Berücksichtigung von Menge und Struktur des Klebers wird es möglich sein, wertvolle Anhaltspunkte über den Backwert des Mehles zu erlangen.

Bei den Arbeiten mit Weizenkleber und Teigen wird das Fehlen eines Verfahrens zur schnellen Bestimmung der Trockensubstanz allgemein als ein Übelstand empfunden und ich glaube, daß auch wegen der schwierigen Austrocknung des Klebers, der oft ergangenen Aufforderung, den Gehalt an Trockenkleber anzugeben, so selten entsprochen wird. Diesem Übelstande hoffe ich bald begegnen zu können; denn ich bin zur Zeit damit beschäftigt, ein Verfahren, welches die Bestimmung der Klebertrockensubstanz schnell und sicher ermöglicht, auszuarbeiten und werde über die erzielten Ergebnisse demnächst in dieser Zeitschrift berichten.

\title{
Über die HaIphen'sche Reaktion.
}

\author{
Von

\section{E. Rupp.}

Mitteilung aus dem Pharmazeutisch-chemischen Institut Marburg.

Die Empfindlichkeit der Halphen'schen Reaktion auf Baumwollsamenöl ist erfahrungsgemäß in hohem Grade von der Art der Ausführung abhängig. Die Reaktionsgemische mögen gleiche Anfangszusammensetzung baben und im gleichen Heizbade erhitzt werden, dennoch weichen die erreichten Farbintensitäten erheblich voneinander ab, je nachdem mit oder ohne Rücklaufvorrichtung für den siedenden Schwefelkohlenstoff gearbeitet wird.

Der positive Reaktionseintritt ist geknüpft an einen gewissen Schwefelkohlenstoffgehalt einerseits, an eine über dem Siedepunkt des Schwefelkohlenstoffs liegende Temperatur andererseits, also zwei einander widerstrebende Faktore, insofern als abfallende Schwefelkohlenstoffkonzentration ein Ansteigen der Temperatur zur Folge hat und umgekehrt. So ist es auch leicht erklärlich, dab selbst bei Anwendung eines Schwefelkohlenstoff-Kondensors noch beträchtliche Unterschiede in den Reaktionsintensitäten sich bemerkbar machen, je nach dessen größerer oder geringerer Wirksamkeit. Ich verweise diesbezüglich auf die unlängst erschienene Arbeit von $K \ddot{u} h n$ und Bengen ${ }^{1}$ ) nebst den darin enthaltenen Literaturauszügen.

Um gleiche Reaktionsstärken zu erhalten, was für praktische Zwecke im Interesse eines gleichen Ausfalls der Halphen'schen Reaktionen erwünscht sein muß, ist es also erforderlich, die beiden Faktoren Schwefelkohlenstoffkonzentration und Reaktionstemperatur dauernd konstant zu erhalten, und zwar bei einer Temperatur, welche dem von Halphen ermittelten Optimum von $100-105^{\circ}$ möglichst nahe kommt. Diesen Bedingungen ist praktisch leicht Genüge zu leisten, indern man die Reaktion in geschlossenem Gefäße bei Siedetemperatur des Wassers sich vollziehen läßt.

1) Diese Zeitschrift 1906, 12, 145. 